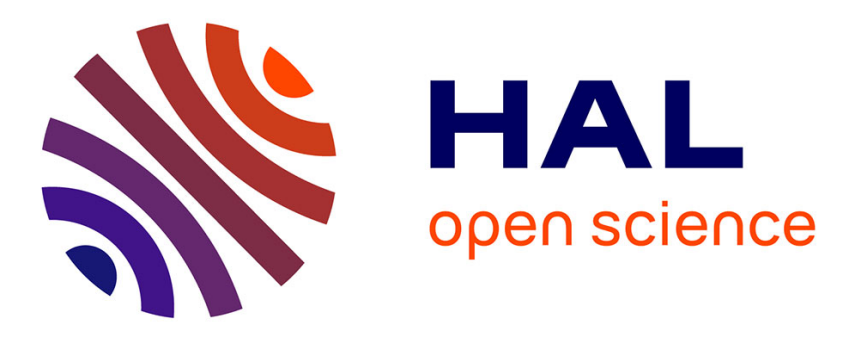

\title{
Two-Dimensional 3He: A Crucial System for Understanding Fermion Dynamics
}

Helga M. Böhm, Eckhard Krotscheck, Martin Panholzer, Henri Godfrin, Hans J. Lauter, Matthias Meschke

\section{- To cite this version:}

Helga M. Böhm, Eckhard Krotscheck, Martin Panholzer, Henri Godfrin, Hans J. Lauter, et al.. TwoDimensional 3He: A Crucial System for Understanding Fermion Dynamics. Journal of Low Temperature Physics, 2010, 158, pp.194. 10.1007/s10909-009-0033-6 . hal-00921348

\section{HAL Id: hal-00921348 https://hal.science/hal-00921348}

Submitted on 20 Dec 2013

HAL is a multi-disciplinary open access archive for the deposit and dissemination of scientific research documents, whether they are published or not. The documents may come from teaching and research institutions in France or abroad, or from public or private research centers.
L'archive ouverte pluridisciplinaire HAL, est destinée au dépôt et à la diffusion de documents scientifiques de niveau recherche, publiés ou non, émanant des établissements d'enseignement et de recherche français ou étrangers, des laboratoires publics ou privés. 


\title{
Two-Dimensional ${ }^{3} \mathrm{He}$ : A Crucial System for Understanding Fermion Dynamics
}

\author{
H. M. Böhm ${ }^{1}$. E. Krotscheck ${ }^{1}$. M. Panholzer ${ }^{1}$. \\ H. Godfrin ${ }^{2}$ - H. J. Lauter ${ }^{3}$ - M. Meschke M $^{2,4}$
}

XX.XX.2009

Keywords $\mathrm{He}^{3}$, Fermi Liquid Theory, Dynamic Response

Abstract Neutron scattering measurements at the Institut Laue-Langevin off quasi-twodimensional ${ }^{3} \mathrm{He}$ have shown, for the first time, a situation where the collective mode crosses the particle-hole continuum and reappears, at a momentum transfer of $q \approx 1.55 \AA^{-1}$ as a well-defined collective excitation.

The effect is well described by the Fermion generalization of multi-particle fluctuation theory of Jackson, Feenberg, and Campbell that has been so successful for bosonic quantum fluids. We describe the theory briefly and state that it can be mapped onto the form of timedependent Hartree-Fock theory (TDHF) containing energy dependent effective interactions; these are obtained from microscopic ground state theory.

Our theoretical result has far-reaching consequences: a popular paradigm in discussing the density-density response function of Fermi systems is the "random phase approximation" (RPA), most frequently applied with some static interaction and, perhaps, some effective mass. Such a "phenomenologically modified" RPA can be justified only under severe simplifying approximations and is unable to describe the experimental situation consistently. As soon as one goes beyond the RPA, intermediate states which cannot be described in terms of the quantum numbers of a single (quasi-)particle become essential for capturing the correct physics. In oder to understand the above mentioned experiment, their appropriate inclusion, as presented in this work, is essential.

PACS numbers: 67.25.-k, 67.25.bh, 67.25.dt

1:Institute for Theoretical Physics, Johannes Kepler University, Austria

E-mail: eckhard.krotscheck@jku.at

2: Institut Néel, CNRS et Université Joseph Fourier, BP 166, F-38042 Grenoble Cedex 9, France

E-mail: henri.godfrin@grenoble.cnrs.fr

3: Institut Laue-Langevin, 38042 Grenoble Cedex, France

E-mail: lauter@ill.fr

4: present address: Low Temperature Laboratory, Helsinki University of Technology P.O. Box 3500, FIN-

02015 TKK, Finland 


\section{Introduction}

Liquid ${ }^{3} \mathrm{He}$ has always been the prime example for a "strongly correlated" Fermi fluid. Compared with other (mostly electronic) so-called "strongly correlated" systems its full understanding poses one of the most demanding challenges for the theorist. Experiments and theoretical discussions of the dynamics of bulk ${ }^{3} \mathrm{He}$ are abundant, but there is very little information of the same system in two dimensions.

A popular language ${ }^{1,2}$ to discuss the the dynamic response of ${ }^{3} \mathrm{He}$ is the "random phase approximation" (RPA) where the density-density response function $\chi(q, \omega)$ is written as

$$
\chi(q, \omega)=\frac{\chi_{0}(q, \omega)}{1-V_{\mathrm{p}-\mathrm{h}}(q) \chi_{0}(q, \omega)},
$$

where $V_{\mathrm{p}-\mathrm{h}}(q)$ is an effective ("particle-hole") interaction, and $\chi_{0}(q, \omega)$ is the densitydensity response function of the non-interacting (Bose or Fermi) system. The effective interaction can be determined from observable data by demanding that the first two energy weighted sum rules are satisfied, i.e.

$$
S(q)=-\int_{0}^{\infty} \frac{d(\hbar \omega)}{\pi} \mathfrak{I} m \chi(q, \omega), \quad \frac{\hbar^{2} q^{2}}{2 m}=-\int_{0}^{\infty} \frac{d(\hbar \omega)}{\pi}(\hbar \omega) \mathfrak{I} m \chi(q, \omega) .
$$

Since the static structure factor is known experimentally, there is no reason for not satisfying these sum rules. In the case of a Bose system, we have

$$
\chi_{0}(q, \omega)=\frac{\frac{\hbar^{2} q^{2}}{m}}{\hbar^{2} \omega^{2}-\left(\frac{\hbar^{2} q^{2}}{2 m}\right)^{2}} .
$$

The procedure outlined above then leads to the famous Feynman dispersion relation ${ }^{3} \hbar \omega(q)=$ $\hbar^{2} q^{2} / 2 m S(q)$.

The RPA has also been reasonably successful in electron structure theory, where it even legitimate to replace the $V_{\mathrm{p}-\mathrm{h}}(q)$ by the Coulomb potential because it (a) is the only interaction describing real particles that has a Fourier transform and (b) the bare Coulomb interaction dominates for long wave lengths.

In a strongly interacting system like ${ }^{3} \mathrm{He}$, the expression (1) also gives, when combined with the sum rules (2) a qualitative description of the situation: a collective mode and a "particle-hole" continuum. Compared with the equally well studied Bose fluid ${ }^{4} \mathrm{He}$, two effects appear to be superimposed: The "phonon-roton" spectrum known from ${ }^{4} \mathrm{He}$, and the "particle-hole continuum" typical for a Fermi fluid. For a discussion of the relevant physics see, for example, the review article by David Pines ${ }^{2}$. In particular it is evident that there should not be a well-defined roton excitation in ${ }^{3} \mathrm{He}$ because the collective mode would be, in the relevant energy/momentum regime, inside the particle-hole continuum.

Upon closer examination, the picture is less satisfactory: Similar to the case of ${ }^{4} \mathrm{He}$, all energies are typically about a factor of two too high. For bosons, the effect has been explained intuitively by "backflow" 4 , and by systematically expanding upon Feynman's ideas we have reached now a very good understanding of the dynamic structure function of interacting bosons in the full $(k, \omega)$ plane.

Pines $^{2}$ points out that "Woods's experiment let me conclude that the phonon-maxonroton excitation in He II and the zero sound mode of ${ }^{3} \mathrm{He}$ had a common physical origin in strong (and quite similar) effective interatomic interactions ...". However, the theoretical 

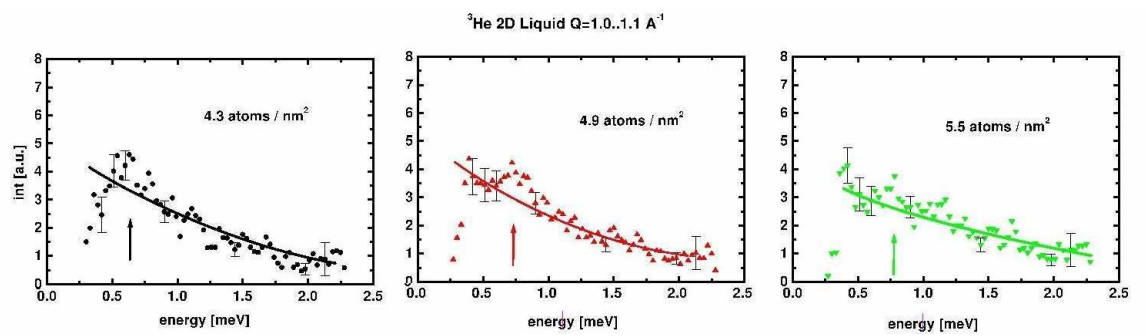

Fig. 1 The figure shows experimental data of the dynamic structure function $S(q, \omega)$ in two-dimensional ${ }^{3} \mathrm{He}$ for three densities as shown in the figure, for wave numbers $1.1 \AA^{-1} \leq q \leq 1.2 \AA^{-1}$. These data were obtained by Godfrin, Lauter, and Meschke at the IN14 instrument at the Institute Laue-Langevin in Grenoble.

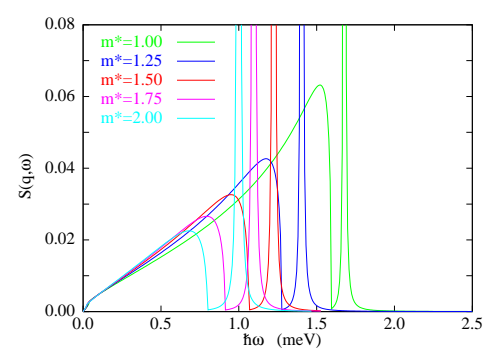

Fig. 2 RPA dynamic structure function $S(q, \omega)$ in $2 \mathrm{D}{ }^{3} \mathrm{He}$ for the momentum transfer $q=1.05 \AA^{-1}$ as a function of an assumed effective mass ratio $1 \leq m^{*} / m \leq 2$. (The collective mode was artificially broadened.)

description of the dynamics of ${ }^{3} \mathrm{He}$ went along different lines: The energy of the collective mode can also be lowered by postulating an effective mass in the particle-hole spectrum. It should be noted that this implies that the position of the collective mode is determined, for bosons and fermions, by totally different mechanism.

\section{$2{ }^{3} \mathrm{He}$ in two dimensions}

Measurements of the dynamic structure function $S(k, \omega)$ of ${ }^{3} \mathrm{He}$ films provide a crucial test for the validity of the general "RPA-like" procedure for lowering the collective mode compared to the RPA prediction. Fig. 1 shows data from the Laue Langevin Institute that have, so far, only been circulated in conference proceedings ${ }^{5}$ and await theoretical explanations.

The collective mode (the mode with maximum strength as indicated by an arrow) is found inside a broad continuum. We demonstrate in Fig. 2 that it is not possible to reconcile this feature with an RPA based response function (1) within the procedure outlined above. Fig. 2 shows theoretical results for $S(k, \omega)$ for $q=1.05 \AA^{-1}$ at an areal density of $n=$ $0.043 \AA^{-2}$ : Trying to adjust the location of the collective mode by introducing an effective mass $m^{*}$ can move the main peak towards the experimental value, however, the very different shape of the calculated spectra and those found experimentally is evident. In particular, the one-particle-one-hole continuum always lies below the collective mode. 


\section{Pair-Excitation Theory}

We have taken the view that the physical mechanisms that determine the energetics of the phonon-roton spectrum are indeed the same in ${ }^{3} \mathrm{He}$ and ${ }^{4} \mathrm{He}$. For bosons, a systematic theory for the dynamics that supersedes Feynman's intuitive "backflow" theory is based on a timedependent, correlated wave function

$$
|\Psi(t)\rangle=\frac{e^{-\mathrm{i} E_{0} t / \hbar} e^{\frac{1}{2} \delta U(t)}\left|\Psi_{0}\right\rangle}{\left[\left\langle\Psi_{0}\left|e^{\Re e \delta U(t)}\right| \Psi_{0}\right\rangle\right]^{1 / 2}}
$$

where $\left|\Psi_{0}\right\rangle$ is the ground-state wavefunction, $E_{0}$ is its energy, and

$$
\delta U(t)=\sum_{i} \delta u^{(1)}\left(\mathbf{r}_{i} ; t\right)+\sum_{i<j} \delta u^{(2)}\left(\mathbf{r}_{i}, \mathbf{r}_{j} ; t\right)+\cdots
$$

is the complex excitation operator. The method has by now been developed up to the threebody order; it provides a consistent description of the dynamics of ${ }^{4} \mathrm{He}$ in the whole $(q, \omega)$ plane. The generalization of this wave function to fermions is to replace the fluctuations $\delta u^{(n)}\left(\mathbf{r}_{1}, \ldots, \mathbf{r}_{n} ; t\right)$ by particle-hole operators, i.e.

$$
U(t)=\sum_{p h} \delta u_{p h}^{(1)}(t) a_{p}^{\dagger} a_{h}+\sum_{p p^{\prime} h h^{\prime}} \delta u_{p p^{\prime} h h^{\prime}}^{(2)}(t) a_{p}^{\dagger} a_{p^{\prime}}^{\dagger} a_{h^{\prime}} a_{h}
$$

The dynamic wave function is then written as

$$
|\Psi(t)\rangle=\frac{1}{\mathscr{N}} e^{-\mathrm{i} E_{0} t / \hbar} F e^{\frac{1}{2} U(t)}\left|\Phi_{0}\right\rangle
$$

where $F$ is a local correlation operator, and $\left|\Phi_{0}\right\rangle$ a model wave function, normally a Slater determinant of plane waves. The derivation of the equations of motion proceeds then along the same lines as for bosons ${ }^{6,7,8}$ but it is, of course, significantly more complicated due to the multitude of exchange diagrams.

The simplest approximation leaves out the pair excitations ${ }^{9}$. In that case, the theory can be mapped onto the form of a time-dependent Hartree-Fock theory, in which the strong, bare interaction is replaced by a weak, effective interaction that is tamed by the correlations ${ }^{10}$. When, in addition, exchange terms are omitted, an response-function of the form (1) is obtained and the procedure for obtaining the excitations corresponds exactly to that outlined in the introduction.

Owing to the argument that the same mechanism determine the energetics of the phononroton spectrum should be at work in ${ }^{4} \mathrm{He}$ and ${ }^{3} \mathrm{He}$, we have used the form (6) for the dynamic wave function. The important aspect here are the pair fluctuations which contribute much of the shift of the roton energy from it's RPA value to the experimental one. Including this type of fluctuations is technically demanding, but the result is quite plausible: The equations of motion can be cast in the form of an "effective time-dependent Hartree-Fock" theory ${ }^{11}$.

$$
\chi(q, \omega)=\left(\rho_{p h, 0} \rho_{0, p h}\right)\left[\Omega+\mathbf{V}_{p-h}(\omega)\right]^{-1}\left(\begin{array}{c}
\rho_{p h, 0} \\
\rho_{0, p h}
\end{array}\right) .
$$

where

$$
\Omega=\left(\begin{array}{cc}
e_{p}-e_{h}-\hbar \omega-\mathrm{i} \eta & 0 \\
0 & e_{p}-e_{h}+\hbar \omega+\mathrm{i} \eta
\end{array}\right)
$$



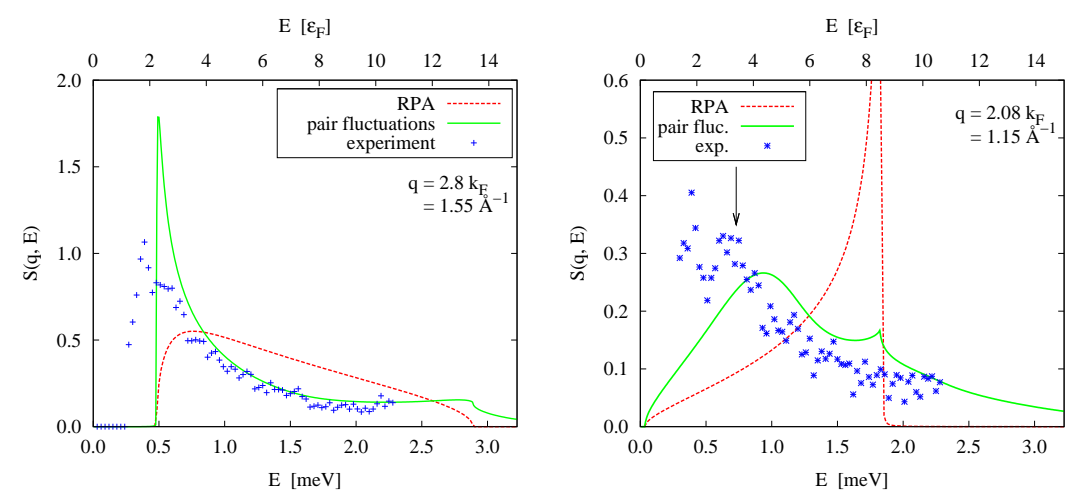

Fig. 3 (color online) The figure shows a comparison of representative experimental and corresponding theoretical data for two different momentum transfers. the red curve is the RPA prediction as defined above, the green curve the prediction of the pair fluctuation theory, and the crosses are experimental data.

where the $e_{k}$ are the single particle energies of correlated basis functions (CBF) theory and $\mathbf{V}_{\mathrm{p}-\mathrm{h}}(\omega)$

$$
\mathbf{V}_{\mathrm{p}-\mathrm{h}}(\omega)=\left(\begin{array}{cc}
V_{p h, p^{\prime} h^{\prime}}^{(\mathrm{A})}(\omega) & V_{p p^{\prime} h h^{\prime}, 0}^{(\mathrm{B})}(\omega) \\
V_{0, p p^{\prime} h h^{\prime}}^{(\mathrm{B})}(-\omega) & V_{p^{\prime} h^{\prime}, p h}^{(\mathrm{A})}(-\omega)
\end{array}\right) .
$$

The interactions are obtained from ground state calculations in terms of Fermi-JastrowFeenberg diagrams ${ }^{12,10}$. We address in a separate paper $^{13}$ the importance of exchange effects; the new aspect introduced by pair fluctuations is that all interaction matrix elements are energy dependent. For the purpose of this paper we distill the physical content of the theory and keep only the direct, local terms, i.e.

$$
V_{p h, p^{\prime} h^{\prime}}^{(\mathrm{A}, \mathrm{B})}(\omega)=\delta_{\mathbf{p}+\mathbf{h}, \mathbf{p}^{\prime}+\mathbf{h}^{\prime}} \tilde{V}^{(\mathrm{A}, \mathrm{B})}(q, \omega)
$$

where $\mathbf{q}=\mathbf{p}-\mathbf{h}$ is the momentum transfer. The $V^{(\mathrm{A})}(q, \omega)$ and $V^{(\mathrm{B})}(q, \omega)$ are normally different. With the generalization of the interaction matrix to energy-dependent quantities, we have in a sense derived the simplest justifiable generalization of the RPA. Note that the density-density response function resulting from this straightforward generalization, and being rather simple in the language of TDHF theory, can not be cast into the form (1). It satisfies, however, the sum rules (2) up to a small error that has to do with the fact that the Jastrow-Feenberg wave function for a Fermi system does not have the correct nodal structure,

Fig. 3 shows the comparison of some experimental data with the results of both the pair fluctuation theory and the RPA for selected momentum transfers. The message is very clear: There is an overall good agreement between experimental data and the predictions of the pair fluctuation theory presented here. In particular, the agreement between experiment and theory at the highest momentum transfer is impressive. There is, on the other hand, no way to understand the experimental data on the basis of an "RPA" formulation (1).

\section{Summary}

It is clear that we are, at this point, to some extent, rewriting "conventional" approaches of many-body physics in condensed matte theory. The "RPA" way to think about the excita- 
tions of a strongly interacting fluid has had enormous impact on the qualitative understanding long-wavelength excitations, but it should be laid to rest as soon as the wave number of the excitation becomes comparable to the interparticle distance. The TDHF theory, much more popular in nuclear physics than in condensed matter theory, has provided the correct framework. The reader is reminded of Richardson's summary talk at the QFS conference in Sanibel in 1983: The history of the subject is interesting because it gives an example of the cross pollination of ideas between research fields. In the 1960 condensed matter theorists applied a great deal of effort to the problem of liquid ${ }^{3} \mathrm{He} . .$. The formal problem was abandoned by condensed matter theorists because it was too difficult for the techniques then available. The question was rediscovered by nuclear theorists after ten years. ... The expertise in the calculations had shifted to a different area of physics, but liquid Helium was available as the model for a nucleus with $10^{23}$ nucleons. We identify, once again, a situation where paradigms, that are the daily bread of a nuclear theorist, provide insight into the mechanisms of other condensed matter systems.

Acknowledgements We thank J. Boronat and his group for supplying quantum Monte Carlo data on the static structure function of two-dimensional ${ }^{3} \mathrm{He}$. This work was supported, in part, by the Austrian Science Fund FWF under project P21264.

\section{References}

1. D. Pines, P. Nozieres, The Theory of Quantum Liquids, vol. I (Benjamin, New York, 1966)

2. D. Pines, Physics Today 34, 106 (1981)

3. R.P. Feynman, Phys. Rev. 94(2), 262 (1954)

4. R.P. Feynman, M. Cohen, Phys. Rev. 102, 1189 (1956)

5. H.M. Böhm, H. Godfrin, E. Krotscheck, H.J. Lauter, M. Meschke, M. Panholzer, International Journal of Modern Physics B 21, 2055 (2007)

6. E. Krotscheck, Phys. Rev. B 31, 4258 (1985)

7. B.E. Clements, E. Krotscheck, C.J. Tymczak, Phys. Rev. B 53, 12253 (1996)

8. C.E. Campbell, E. Krotscheck. Dymanic many body correlations I: Pair fluctutions in bulk ${ }^{4} \mathrm{He}$ (2009). Submitted to Phys. Rev. B

9. J.M.C. Chen, J.W. Clark, D.G. Sandler, Z. Physik A 305, 223 (1982)

10. E. Krotscheck, Phys. Rev. A 26, 3536 (1982)

11. D.J. Thouless, The quantum mechanics of many-body systems, 2nd edn. (Academic Press, New York, 1972)

12. E. Krotscheck, J.W. Clark, Nucl. Phys. A 328, 73 (1979)

13. M. Panholzer, H.M. Böhm, R. Holler, E. Krotscheck. Exchange effects and the dynamics of ${ }^{3}$ he (2009). These proceedings 